\title{
An elementary proof of Fermat-Wiles and Catalan-Mihailescu theorems and generalization to Beal conjecture
}

\section{Jamel Ghanouchi}

\section{RIME department of Mathematics}

Keywords : Diophantine ; Fermat ; Catalan ; Fermat-Catalan ; Resolution.

Abstract: We begin with Fermat, Catlan and Fermat-Catalan equations and solve them.

\section{The Fermat equation}

Fermat equation is $y^{n}=x^{n} \pm z^{n}=x^{n}+a z^{n}$

Let $x^{n-2} y^{2}-y^{n-2} x^{2}=A a$

If $A=0 \Rightarrow x^{n-4}=y^{n-4}$ but $G C D(x, y)=1 \Rightarrow n=4$ impossible, there is no ution

If $A^{2}=z^{2 n} ; n \geq 3 \Rightarrow x^{n-3} y-y^{n-2}=\frac{A a z^{n}}{x} \in Z$ impossible $\Rightarrow n=2$

We have

$\left(x^{n-2} y^{2}-y^{n-2} x^{2}\right) z^{c}=A a z^{n}=A y^{n-2} y^{2}-A x^{n-2} x^{2} \Rightarrow\left(x^{n-2} z^{n}-A y^{n}\right.$

We have then four cases :

$x^{2}=u\left(-x^{n-2} z^{n}+A y^{n-2}\right) ; y^{2}=u\left(-y^{n-2} z^{n}+A x^{n-2}\right)$

$x^{n-2}=v\left(x^{2} z^{n}+A y^{2}\right) ; y^{n-2}=v\left(y^{2} z^{n}+A x^{2}\right)$

or

$u x^{2}=-x^{n-2} z^{n}+A y^{n-2} ; u y^{2}=-y^{n-2} z^{n}+A x^{n-2}$

$v x^{n-2}=x^{2} z^{n}+A y^{2} ; v y^{n-2}=y^{2} z^{n}+A x^{2}$

or

$x^{2}=u\left(-x^{n-2} z^{n}+A y^{n-2}\right) ; y^{2}=u(-y)$

$v x^{n-2}=x^{2} z^{n}+A y^{2} ; v y^{n-2}=y^{2}{ }^{n}+A x$

or

$u x^{2}=-x^{n-2} z^{n}+A y^{n-2}: \ln =-x^{n}+A x^{n} z^{2}$

$x^{n-2}=v\left(x^{2} z^{n}+A y^{2}, y=v\left(y^{2} z, \mathcal{K}^{2}\right)\right.$

With $u, v \in Z$

First case

or

$x^{2}=u_{(n-2}{ }^{n-2}, y^{n-2}=u\left(-y^{n-2} z^{n}+A x^{n-2}\right)$

$x^{n-2}=v(x+A y), y^{n-2}=v\left(y^{2} z^{n}+A x^{2}\right)$

We have

$y^{n}=u v\left(-y^{n} z^{2 n}+A^{2} x^{n}-A z^{n}\left(x^{2} y^{n-2}-y^{2} x^{n-2}\right)\right)=u v\left(-y^{n} z^{2 n}+A^{2} x^{n}+A^{2} a z^{n}\right)=u v\left(A^{2}-z^{2 n}\right) y^{n}$

$u v\left(A^{2}-z^{2 n}\right)=1$

Impossible because $\mathrm{A}, \mathrm{u}, \mathrm{v}$ are integers

Second case

or

$u x^{2}=-x^{n-2} z^{n}+A y^{n-2} ; u y^{2}=-y^{n-2} z^{n}+A x^{n-2}$

$v x^{n-2}=x^{2} z^{n}+A y^{2} ; v y^{n-2}=y^{2} z^{n}+A x^{2}$ 
We have

$u v y^{n}=-y^{n} z^{2 n}+A^{2} x^{n}-A z^{n}\left(x^{2} y^{n-2}-y^{2} x^{n-2}\right)=-y^{n} z^{2 n}+A^{2} x^{n}+A^{2} a z^{n}=\left(A^{2}-z^{2 n}\right) y^{n}$

$u v=A^{2}-z^{2 n}$

And

$u v\left(y^{2} x^{n-2}-x^{2} y^{n-2}\right)=u v A=u z^{2 n}\left(-y^{2 n-4}+x^{2 n-4}\right) A=v z^{2 n}\left(-y^{4}+x^{4}\right) A$

$\Rightarrow u=z^{2 n}\left(-y^{4}+x^{4}\right) ; v=z^{2 n}\left(-y^{2 n-4}+x^{2 n-4}\right)$

$u v=A^{2}-z^{2 n}=\left(y^{4}-x^{4}\right)\left(y^{2 n-4}-x^{2 n-4}\right)=u v z^{-4 n} \Rightarrow u v=A^{2}-z^{2 n}=0$

It is impossible $\Rightarrow n=2$

Third case

or

$x^{2}=u\left(-x^{n-2} z^{n}+A y^{n-2}\right) ; y^{2}=u\left(-y^{n-2} z^{n}+A x^{n-2}\right)$

$v x^{n-2}=x^{2} z^{n}+A y^{2} ; v y^{n-2}=y^{2} z^{n}+A x^{2}$

We have

$v y^{n}=u\left(-y^{n} z^{2 n}+A^{2} x^{n}-A z^{n}\left(x^{2} y^{n-2}-y^{2} x^{n-2}\right)\right)=u\left(-y^{n} z^{2 n}+A^{2} x^{n}\right.$ $v=u\left(A^{2}-z^{2 n}\right)$

And

$v\left(y^{2} x^{n-2}-x^{2} y^{n-2}\right)=v A=u v z^{2 n}\left(-y^{2 n-4}+x^{2 n-4}\right) A=z^{2 n}\left(-4+x^{4}\right) A$

$\Rightarrow u z^{2 n}\left(-y^{2 n-4}+x^{2 n-4}\right)=1 \Rightarrow u=\infty ; n=2$

Impossible because $\mathrm{u}, \mathrm{A}$ are integers

Fourth case

or

$u x^{2}=-x^{n-2} z^{n}+A y^{n-2} ; u y^{2}=-y^{n-2} z^{n}+A$
$x^{n-2}=v\left(x^{2} z^{n}+A y^{2}\right) ; y^{n-2}=v\left(y^{2}\right.$

We have

$u y^{n}=v\left(-y^{n} z^{2 n}+A^{2} x^{n}-\left(z^{n}\left(x-2-y^{2} x^{n}\right)\right)=v\left(-y^{n} z^{2 n}+A^{2} x^{n}+A^{2} a z^{n}\right)=v\left(A^{2}-z^{2 n}\right) y^{n}\right.$
$u=v\left(A^{2}-z^{2 n}\right)$

And

$u\left(y^{2} x^{n-2}-x^{2} v^{n-2}\right)=\quad=z^{2 n}\left(-y^{2 n-4}+x^{2 n-4}\right) A=u v z^{2 n}\left(-y^{4}+x^{4}\right) A$

$\Rightarrow v z^{2 n}(-)=1 \Rightarrow, x^{4}=y^{4}+1$

Imposs, $\mathrm{A}$ are integers !

The only tion is $\mathrm{A}=1$ and $\mathrm{n}=2$

\section{Resolution of Catalan equation}

Catalan equation is $y^{p}=x^{q}+1$

Let $x^{q-3} y^{2}-y^{p-2} x^{3}=1$

If $A=0 \Rightarrow x^{q-6}=y^{p-4}$

$G C D(x, y)=1 \Rightarrow p=4$ impossible, there is no solution, Ko Chao proved it and

If $A^{2}=1 ; p \geq 3 \Rightarrow x^{q-3} y-y^{p-3} x^{3}=1 / \mathrm{y} \in Z$ impossible thus $\mathrm{p}=2$

We have

$x^{q-3} y^{2}-y^{p-2} x^{3}=1=A y^{p-2} y^{2}-A x^{q-3} x^{3}$ 
$\Rightarrow\left(x^{q-3}-A y^{p-2}\right) y^{2}=\left(y^{p-2}-A x^{q-3}\right) x^{3}$

$\left(y^{2}+A x^{3}\right) x^{q-3}=\left(x^{3}+A y^{2}\right) y^{p-2}$

$G C D(x, y)=1$

We have then four cases :

$x^{3}=u\left(-x^{q-3}+A y^{p-2}\right) ; y^{2}=u\left(-y^{p-2}+A x^{q-3}\right)$

$x^{q-3}=v\left(x^{3}+A y^{2}\right) ; y^{p-2}=v\left(y^{2}+A x^{3}\right)$

or

$u x^{3}=-x^{q-3}+A y^{p-2} ; u y^{2}=-y^{p-2}+A x^{q-3}$

$v x^{q-3}=x^{3}+A y^{2} ; v y^{p-2}=y^{2}+A x^{3}$

or

$x^{3}=u\left(-x^{q-3}+A y^{p-2}\right) ; y^{2}=u\left(-y^{p-2}+A x^{q-3}\right)$

$v x^{q-3}=x^{3}+A y^{2} ; v y^{p-2}=y^{2}+A x^{3}$

or

$u x^{3}=-x^{q-3}+A y^{p-2} ; u y^{2}=-y^{p-2}+A x^{q-3}$

$x^{q-3}=v\left(x^{3}+A y^{2}\right) ; y^{p-2}=v\left(y^{2}+A x^{3}\right)$

$u, v \in Z$

First case

$x^{3}=u\left(-x^{q-3}+A y^{p-2}\right) ; y^{2}=u\left(-y^{p-2}+A x^{q-3}\right)$

$x^{q-3}=v\left(x^{3}+A y^{2}\right) ; y^{p-2}=v\left(y^{2}+A x^{3}\right)$

We have

$y^{p}=u v\left(-y^{p}+A^{2} x^{q}-A\left(x^{3} y^{p-2}-y^{2} x^{q-3}\right)\right)=u v\left(-1+1 x A^{2}\right)=u v\left(A^{2}-1\right) y^{n}$

$u v\left(A^{2}-1\right)=1$

Impossible because $\mathrm{A}, \mathrm{u}, \mathrm{v}$ are inte $\mathrm{rs}$

Second case

$u x^{3}=-x^{q-3}+A y^{p-2} ; u y^{2}={ }^{2-2}+A x^{q}$

$v x^{q-3}=x^{2}+A y^{2} ; v y^{p-2}$

We have

$u v y^{p}=-y^{p}+A\left(y^{p-2}-y^{2} x^{q-3}\right)=-y^{p}+A^{2} x^{q}+A^{2}=\left(A^{2}-1\right) y^{p}$

$u v=A^{2}-1$

And

$u v\left(y^{2}-x^{3} \cdot{ }^{p-2}\right)=u v A=u\left(-y^{2 p-4}+x^{2 q-6}\right) A=v\left(-y^{4}+x^{6}\right) A$

$\Rightarrow u=-y \quad x^{0} ; v=-y^{2 p-4}+x^{2 q-6}$

$\left.u v=A^{2}-1=x^{4}\right)\left(y^{2 p-4}-x^{2 q-6}\right)$

$\Rightarrow\left(y^{2} x^{q-3}-x^{3} y^{p-2}\right)^{2}-1=\left(y^{4}-x^{6}\right)\left(y^{2 p-4}-x^{2 q-6}\right)$

$\Rightarrow x^{2 q}+y^{2 p}+2 x^{q} y^{p}=2 y^{4} x^{2 q-6}+2 x^{6} y^{2 p-4}-1$

$=\left(x^{q}+y^{p}\right)^{2}=\left(2 y^{p}-1\right)^{2}=4 y^{2 p}-4 y^{p}+1$

$2 p>4 \Rightarrow 2 y^{3} x^{2 q-6}+2 x^{6} y^{2 p-5}-4 y^{2 p-1}+4 y^{p-1}=2 / \mathrm{y} \in Z$

Impossible : $\Rightarrow p=2$

Third case

$x^{3}=u\left(-x^{q-3}+A y^{p-2}\right) ; y^{2}=u\left(-y^{p-2}+A x^{q-3}\right)$

$v x^{q-3}=x^{3}+A y^{2} ; v y^{p-2}=y^{2}+A x^{3}$ 
We have

$v y^{p}=u\left(-y^{p}+A^{2} x^{q}-A\left(x^{3} y^{p-2}-y^{2} x^{q-3}\right)\right)=u\left(-y^{p}+A^{2} x^{q}+A^{2}\right)=u\left(A^{2}-1\right) y^{p}$

$v=u\left(A^{2}-1\right)$

And

$v\left(y^{2} x^{q-3}-x^{3} y^{p-2}\right)=v A=u v\left(-y^{2 p-4}+x^{2 q-6}\right) A=\left(-y^{4}+x^{6}\right) A \Rightarrow u\left(-y^{2 p-4}+x^{2 q-6}\right)=1 \Rightarrow p-2=q-3=$ 0

Impossible because $\mathrm{u}, \mathrm{A}$ are integers

Fourth case

$u x^{3}=-x^{q-3}+A y^{p-2} ; u y^{2}=-y^{p-2}+A x^{q-3} x^{q-3}=v\left(x^{3}+A y^{2}\right) ;$

$y^{p-2}=v\left(y^{2}+A x^{3}\right)$

We have

$u y^{p}=v\left(-y^{p}+A^{2} x^{q}-A\left(x^{2} y^{p-2}-y^{2} x^{q-3}\right)\right)=v\left(-y^{p}+A^{2} x^{q}+A^{2}\right)=v\left(A^{2}-1\right) y^{p}$

$u=v\left(A^{2}-1\right)$

And

$u\left(y^{2} x^{q-3}-x^{3} y^{p-2}\right)=u A=\left(-y^{2 p-4}+x^{2 q-6}\right) A=u v\left(-y^{4}+x^{6}\right) A \Rightarrow v\left(-y^{4}+x^{0}\right)=1$

Impossible, because $\mathrm{v}, \mathrm{A}$ are integers !

The only solution is $\mathrm{A}=1$ and $\mathrm{p}=2$ this case has been studied

\section{The Fermat-Catalan equation}

The equation now is $y^{p}=x^{q} \pm z^{c}=x^{q}+a z^{c}$

Let $x^{q-w} y^{2}-y^{p-2} x^{w}=A$

If

$A=0 \Rightarrow x^{q-2 w}=y^{p-4}$

$G C D(x, y)=1 \Rightarrow p=4$

It means that $\mathrm{p}=2$ is the prime sol on !

And

$A^{2}=z^{2 c} ; p \geq 3 \Rightarrow x^{q-w} y$

impossible thus $\mathrm{p}$

We have

$\left(x^{q-w} y^{2}-y^{p-2} x^{w}\right) \quad A a z^{c}=y^{p-2} y^{2}-A x^{q-w} x^{w}$

$\Rightarrow\left(x^{q-w} z^{c},{ }^{p-2}\right) y \quad\left(y^{p-2} z^{c}-A x^{q-w}\right) x^{2}$

$\left.x^{q-w} y^{2} \quad y^{p-2}\right)=a A z^{c} \quad A y^{p-2} y^{2}-A x^{q-w} x^{w}$

$\Rightarrow\left(x^{q} \quad 4 y^{p-2}-A x^{q-w}\right) x^{w}$

$\left(y^{2}+A x^{n}\right)=\left(x^{w}+A y^{2}\right) y^{p-2}$

$\operatorname{GCD}(x, y)=$

We have then four cases :

First case

$x^{w}=u\left(-x^{q-w} z^{c}+A y^{p-2}\right) ; y^{2}=u\left(-y^{p-2} z^{c}+A x^{q-w}\right)$

$x^{q-w}=v\left(x^{w} z^{c}+A y^{2}\right) ; y^{p-2}=v\left(y^{2} z^{c}+A x^{w}\right)$

We have

$y^{p}=u v\left(-y^{p} z^{2 c}+A^{2} x^{q}-A z^{c}\left(x^{w} y^{p-2}-y^{2} x^{q-w}\right)\right)=u v\left(-y^{p} z^{2 c}+A^{2} x^{q}+A^{2} a z^{c}\right)=u v\left(A^{2}-z^{2 c}\right) y^{p}$

$u v\left(A^{2}-z^{2 c}\right)=1$ 
Impossible because $\mathrm{A}, \mathrm{u}, \mathrm{v}$ are integers

Second case

$u x^{w}=-x^{q-w} z^{c}+A y^{p-2} ; u y^{2}=-y^{p-2} z^{c}+A x^{q-w}$

$v x^{q-w}=x^{w} z^{c}+A y^{2} ; v y^{p-2}=y^{2} z^{c}+A x^{w}$

We have

$\left.u v y^{p}=-y^{p} z^{2 c}+A^{2} x^{q}-A z^{c}\left(x^{w} y^{p-2}-y^{2} x^{q-w}\right)=-y^{p} z^{2 c}+A^{2} x^{q}+A^{2} a z^{c}\right)=\left(A^{2}-z^{2 c}\right) y^{p}$

$u v=A^{2}-z^{2 c}$

And

$u v\left(y^{2} x^{q-w}-x^{w} y^{p-2}\right)=u v A=u\left(-y^{2 p-4}+x^{2 q-2 w}\right) A=v\left(-y^{4}+x^{2 w}\right) A$

$\Rightarrow u=-y^{4}+x^{2 w} ; v=-y^{2 p-4}+x^{2 q-2 w}$

$u v=A^{2}-z^{2 c}=\left(y^{4}-x^{2 w}\right)\left(y^{2 p-4}-x^{2 q-2 w}\right)=u v z^{4 c} \Rightarrow u v=A^{2}-z^{2 c}=0$

Impossible : $\Rightarrow p=2$

Third case

$x^{w}=u\left(-x^{q-w} z^{c}+A y^{p-2}\right) ; y^{2}=u\left(-y^{p-2} z^{c}+A x^{q-w}\right)$

$v x^{q-w}=x^{w} z^{c}+A y^{2} ; v y^{p-2}=y^{2} z^{c}+A x^{w}$

We have

$v y^{p}=u\left(-y^{p} z^{2 c}+A^{2} x^{q}-A z^{c}\left(x^{w} y^{p-2}-y^{2} x^{q-w}\right)\right)=u\left(-y^{p} z^{2}\right.$

$v=u\left(A^{2}-z^{2 c}\right)$

And

$v\left(y^{2} x^{q-w}-x^{w} y^{p-2}\right)=v A=u v\left(-y^{2 p-4}+x^{2 q-2 w}\right) A=\left(-y^{4}+\lambda\right.$

$\Rightarrow u\left(-y^{2 p-4}+x^{2 q-2 w}\right)=1 \Rightarrow p=2$

Impossible because $\mathrm{u}, \mathrm{A}$ are integers

Fourth case

$u x^{w}=-x^{q-w} z^{c}+A y^{p-2} ; u y^{2}=-y$

$x^{q-w}=v\left(x^{w} z^{c}+A y^{2}\right) ; y^{p-2}=v($

We have

$u y^{p}=v\left(-y^{p} z^{2 c}+A^{2} x^{q}-1 z^{c}\left(x-2-y^{2} x^{A-w}\right)\right)=v\left(-y^{p} z^{2 c}+A^{2} x^{q}+A^{2} a z^{c}\right)=v\left(A^{2}-z^{2 c}\right) y^{p}$
$u=v\left(A^{2}-z^{2 c}\right)$

And

$u\left(y^{2} x^{q-w}-w^{w} v^{p-2}\right)=-y^{-4}+x^{2 q-2 w} A=u v\left(-y^{4}+x^{2 w}\right) A$

$\Rightarrow v(-y+x)=1$

Imposs,$A$ are integers ! In the Fermat-Catalan equation, one of the exponents must be equal to The Beal conjecture has been proved!

In fact, in the ee precedent equations studied here, one of the exponent greater or equal to 2 must be minimum, which means that it must be 2 !

\section{Conclusion}

We have solved both three equations by the same method and proved two theorems and one conjecture.

\section{References}

[1] Paolo Ribenboïm, The Catalan's conjecture, Academic Press, 1994.

[2] Robert Tijdeman, On the equation of Catalan, Acta Arith, 1976. 